\title{
Estimating coefficient bounds with respect to a generalized starlike functions on symmetric points
}

\author{
R. Ambrose Prabhu ${ }^{1 *}$ S. Bhaskaran²
}

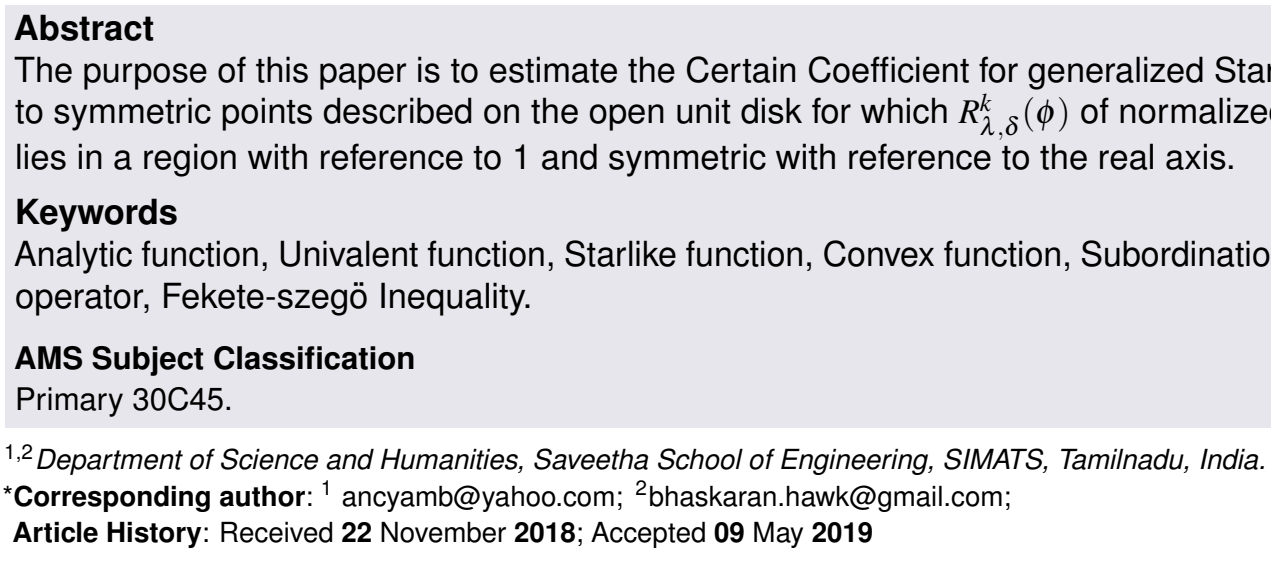

\section{Contents}

1 Introduction 243

2 ete-szegö Problem for the Function of the class

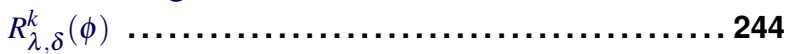

3 Applications to Analytic Function Defined by Frac-

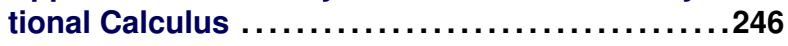

References ............................. 248

\section{Introduction}

Let $\mathscr{A}$ symbolize the class of all analytic function $f(z)$ as concerns to

$$
f(z)=z+\sum_{n=2}^{\infty} a_{n} z^{n}
$$

that are analytic in $\mathbb{U}=\{z \in \mathbb{C}:|z|<1\}$ and agree the conditions $f(0)=0, f^{\prime}(0)=1$. Also $\mathscr{S}$ the subclass of $\mathscr{A}$ consisting of all functions that are univalent in $\mathbb{U}$. For $f(z)$ and $g(z)$ analytic in $\mathbb{U}, f(z)$ is said to subordinate to $g(z)$ when there exist a schwarz function $\omega(z)$, analytic in $\mathbb{U}$ amidst

$$
\omega(0)=0 \quad \text { and } \quad|\omega(z)|<1 \quad(z \in \mathbb{U}),
$$

satisfying

$$
f(z)=g(\omega(z)) \quad(z \in \mathbb{U}) .
$$

This subordination is symbolized as

$$
f \prec g \quad \text { or } \quad f(z) \prec g(z) \quad(z \in \mathbb{U}) .
$$

More precisely, when $g(z)$ is univalent in $\mathbb{U}$, then the subordination be correspondent to

$$
f(0)=g(0) \quad \text { and } \quad f(\mathbb{U}) \subset g(\mathbb{U}) .
$$

Assume $\phi(z)$ an analytic function in $\mathbb{U}$ with $\phi(0)=1, \phi^{\prime}(0)>$ 0 and $\operatorname{Re}\{\phi(z)\}>0, z \in \mathbb{U}$ that map $\mathbb{U}$ onto a starlike region with referrence to 1 and symmetric amidst the real axis. We signify $S^{*}(\phi)$ and $C(\phi)$, respectively, the subclasses of $\mathscr{A}$, that accomplish the relations of subordination:

$$
\frac{z f^{\prime}(z)}{f(z)} \prec \phi(z), \quad z \in \mathbb{U}
$$

and

$$
1+\frac{z f^{\prime \prime}(z)}{f^{\prime}(z)} \prec \phi(z), z \in \mathbb{U} .
$$

The above functions were put forward and studied by Ma and Minda[9]. Specifically, while

$$
\phi(z)=\frac{1+(1-2 \alpha) z}{1-z}, z \in \mathbb{U}, 0 \leq \alpha<1,
$$

these functions diminish respectively to the established classes $S^{*}(\alpha),(0 \leq \alpha<1)$ of $\alpha$ in $\mathbb{U}$ and $C(\alpha),(0 \leq \alpha<1) \alpha$ in U. Ma and Minda [9], the Fekete-Szegö inequality for $f(z)$ 
in $C(\phi)$ was acknowledged in relating the classes $S^{*}(\phi)$ and $C(\phi)$ of Alexander result.

To recollect the Fekete-Szegö problems in consideration to starlike, convex including numerous subclasses in $\mathscr{A}$, the readers are advised to refer the work carried out by Srivatsava et al [20]. Moreover, the primary outcome be oblidged to Fekete and szegö [2] in 1933. Almost 30 years later, Keogh and Merkes [4] derived the problem considering certain subclasses of univalent functions. These articles $[\mathbf{2 , 4 , 6 , 7 ]}$ gave remarkable results that are used to solve problems for other extended classes. Thereafter, Shanmugam et al [17] contributed the Fekete-Szegö problem on view of subclasses of starlike functions up on symmetric points. Inspiring by the above work, we derive the Fekete-Szegö inequality in Theorem 2.1 given below, being extended to prevailing class of normalized analytic functions.

While $\lambda, \delta \in N, k \in N_{0}$ Darus [1] put forward the operator $D_{\lambda, \delta}^{k}$ characterized as

$$
D_{\lambda, \delta}^{k} f(z)=z+\sum_{n=2}^{\infty}[1+(n-1) \lambda]^{k} C(\delta, n) a_{n} z^{n}
$$

In this paper, we attain the Fekete-szegö inequality considering the function $f \in \mathscr{A}$ in the class $R_{\lambda, \delta}^{k}(\phi)$ characterized as follows

Definition 1.1. Let $D_{\lambda, \delta}^{k}: \mathscr{A} \longrightarrow \mathscr{A}$ is a linear operator and $D_{\lambda, \delta}^{k}$ is analytic in $f(U)$.

Let

$$
D_{\lambda, \delta}^{k} f(z)=z+\sum_{n=2}^{\infty}[1+(n-1) \lambda]^{k} C(\delta, n) a_{n} z^{n}
$$

where

$$
C(\delta, n)=\frac{\Gamma(n+\delta)}{\Gamma(n) \quad \Gamma(\delta+1)}
$$

while $\lambda=1$ and $\delta=0$ sălăgean differential operator is obtained. when $k=0$ or $\lambda=0$ leads to Ruscheweyh operator, also $\delta=0$ leads to Al-oboudi differential operator with order $k$

$$
D_{1,0}^{0} f(z)=f(z), D_{1,0}^{1} f(z)=z f^{\prime}(z)
$$

Definition 1.2. An univalent starlike function $\phi(z)$ with referrence to 1 that maps $\mathbb{U}$ onto the right half plane that is symmetric with referrence to the real axis $\phi(0)=1$ and $\phi^{\prime}(0)>1 . f \in \mathscr{A}$ that belongs to the class $R_{\lambda, \delta}^{k}(\phi)$ if

$$
\frac{(s-t) z\left[D_{\lambda, \delta}^{k} f(z)\right]^{\prime}}{D_{\lambda, \delta}^{k}[f(s z)]-D_{\lambda, \delta}^{k}[f(t z)]} \prec \phi(z), \quad\left(\lambda, \delta \in \mathbb{N}, k \in \mathbb{N}_{0}\right) .
$$

In order to establish our important results, we require the subsequent lemma.
Lemma 1.3. [9] An analytic function $p_{1}(z)=1+c_{1} z+c_{2} z^{2}+$ ... with positive real part in $\mathbb{U}$, then

$$
\left|c_{2}-v c_{1}^{2}\right| \leq \begin{cases}-4 v+2, & \text { if } v \leq 0 \\ 2, & 0 \leq v \leq 1 \\ 4 v-2, & v \geq 1\end{cases}
$$

while $v<0$ or $v>1$, the equality satisfies iff $p_{1}(z)=\frac{1+z}{1-z}$ or one of its rotations. When $0<v<1$, the equality satisfies iff $p_{1}(z)=\frac{1+z^{2}}{1-z^{2}}$ or one of its rotation.

When $v=0$, the equality satisfies iff

$p_{1}(z)=\left(\frac{1}{2}+\frac{1}{2} \gamma\right) \frac{1+z}{1-z}+\left(\frac{1}{2}-\frac{1}{2} \gamma\right) \frac{1-z}{1+z} \quad(0 \leq \gamma \leq 1)$

or one of its rotations. When $v=1$, the equality satisfies iff $p_{1}$ is the reciprocal of one of the functions where the equality satisfies if $v=0$. Moreover, the upper bound is sharp, the same can be improvised as follows, if $0<v<1$ :

$$
\begin{aligned}
\left|c_{2}-v c_{1}^{2}\right|+v\left|c_{1}^{2}\right| & \leq 2,0<v \leq \frac{1}{2} \\
\text { and } \quad\left|c_{2}-v c_{1}^{2}\right|+(1-v)\left|c_{1}^{2}\right| & \leq 2, \frac{1}{2}<v \leq 1
\end{aligned}
$$

The following result is more important forour enquiry.

Lemma 1.4. [15] If $p_{1}(z)=1+c_{1} z+c_{2} z^{2}+\ldots$ is a function with positive real part in $\mathbb{U}$, then

$$
\left|c_{2}-v c_{1}^{2}\right| \leq 2 . \max (1,|2 v-1|) \text {. }
$$

The conclusion is sharp for $p_{1}(z)$ given by

$$
p_{1}(z)=\frac{1+z^{2}}{1-z^{2}}
$$

and

$$
p_{1}(z)=\frac{1+z}{1-z}
$$

\section{Fekete-szegö Problem for the Function of the class $R_{\lambda, \delta}^{k}(\phi)$}

Using Lemma 1.2, Fekete-szegö Problem for the class $R_{\lambda, \delta}^{k}(\phi)$ can be proved.

Theorem 2.1. Let $\phi(z)=1+B_{1} z+B_{2} z^{2}+\cdots$ If $f(z)$ given by (1.1) belongs to the class $R_{\lambda, \delta}^{k}(\phi)$, then

$$
\left|a_{3}-\mu a_{2}^{2}\right| \leq \begin{cases}\Lambda, & \mu \leq \sigma_{1} \\ \eta, & \sigma_{1} \leq \mu \leq \sigma_{2} \\ -\Lambda, & \mu \geq \sigma_{3}\end{cases}
$$


where

$$
\begin{aligned}
& \sigma_{1}=\frac{(\delta+1)(s+t-2)(1+\lambda)^{2 k}}{2(\delta+2)(1+2 \lambda)^{k}\left[\left(s^{2}+s t+t^{2}\right)-3\right]} \\
& {\left[\frac{2\left(B_{2}-B_{1}\right)(s+t-2)-B_{1}^{2}(s+t)}{B_{1}^{2}}\right] \text {, }} \\
& \sigma_{2}=\frac{(\delta+1)(s+t-2)(1+\lambda)^{2 k}}{2(\delta+2)(1+2 \lambda)^{k}\left[\left(s^{2}+s t+t^{2}\right)-3\right]} \\
& {\left[\frac{2 B_{2}(s+t-2)-(s+t) B_{1}^{2}}{B_{1}^{2}}\right] \text {, }} \\
& \sigma_{3}=\frac{(\delta+1)(s+t-2)(1+\lambda)^{2 k}}{2(\delta+2)(1+2 \lambda)^{k}\left[\left(s^{2}+s t+t^{2}\right)-3\right]} \\
& {\left[\frac{\left(2 B_{2}-B_{1}\right)(s+t-2)-B_{1}^{2}(s+t)}{B_{1}^{2}}\right] \text {, }} \\
& \Lambda=\frac{4(\delta+1)(\delta+2)(1+2 \lambda)^{k}\left[3-\left(s^{2}+s t+t^{2}\right)\right]}{B_{1}^{2}} \\
& \times\left[\left(B_{2}-B_{1}^{2}\right)-\frac{B_{1}^{2}}{2}\left(\frac{s+t}{s+t-2}\right.\right. \\
& \left.\left.+\frac{2 \mu(1+2 \lambda)^{k}(\delta+2)\left[\left(s^{2}+s t+t^{2}\right)-3\right]}{(\delta+1)(1+\lambda)^{2 k}(2-s-t)^{2}}\right)\right], \\
& \eta=\frac{2 B_{1}}{(1+2 \lambda)^{k}(\delta+1)(\delta+2)\left(3-\left[s^{2}+s t+t^{2}\right]\right)} \text {. }
\end{aligned}
$$

Further,

If $\sigma_{1} \leq \mu \leq \sigma_{3}$, then

$$
\begin{aligned}
& \left|a_{3}-\mu a_{2}^{2}\right|+\frac{(\delta+1)(s+t-2)(1+\lambda)^{2 k}}{(\delta+2)(1+2 \lambda)^{k}\left[\left(s^{2}+s t+t^{2}\right)-3\right] B_{1}^{2}} \\
& \left\{\left(B_{1}-B_{2}\right)(s+t-2)+\sigma_{4} B_{1}^{2}\right\}\left|a_{2}\right|^{2} \leq \eta .
\end{aligned}
$$

If $\sigma_{3} \leq \mu \leq \sigma_{2}$, then

$$
\begin{aligned}
& \left|a_{3}-\mu a_{2}^{2}\right|+\frac{(\delta+1)(s+t-2)(1+\lambda)^{2 k}}{(\delta+2)(1+2 \lambda)^{k}\left[\left(s^{2}+s t+t^{2}\right)-3\right] B_{1}^{2}} \\
& \left\{B_{2}(s+t-2)-\sigma_{4} B_{1}^{2}\right\}\left|a_{2}\right|^{2} \leq \eta .
\end{aligned}
$$

Where

$$
\begin{aligned}
\sigma_{4}= & {\left[\frac{(s+t)(s+t-2)(\delta+1)(1+\lambda)^{2 k}}{(\delta+1)(s+t-2)(1+\lambda)^{2 k}}+\right.} \\
& \left.\frac{\mu(\delta+2)(1+2 \lambda)^{k}\left[\left(s^{2}+s t+t^{2}\right)-3\right]}{(\delta+1)(s+t-2)(1+\lambda)^{2 k}}\right] .
\end{aligned}
$$

The result is sharp.

Proof. When $f \in R_{\lambda, \delta}^{k}(\phi)$, there shall exist a Schwarz function $w(z)$, analytic in $\mathbb{U}$ with $w(0)=0$ and $|w(z)|<1$ in $\mathbb{U}$ satisfying

$$
\frac{(s-t) z\left[D_{\lambda, \delta}^{k} f(z)\right]^{\prime}}{D_{\lambda, \delta}^{k}[f(s z)]-D_{\lambda, \delta}^{k}[f(t z)]}=\phi(w(z))
$$

A function $p_{1}(z)$ is defined as

$$
p_{1}(z)=\frac{1+w(z)}{1-w(z)} .
$$

since $w(z)$ is a Schwarz function, it is known that $\operatorname{Re}\left\{p_{1}(z)\right\}>$ 0 and $p_{1}(0)=0$. A function $p(z)$ is defined by

$$
\begin{aligned}
p(z) & =\frac{(s-t) z\left[D_{\lambda, \delta}^{k} f(z)\right]^{\prime}}{D_{\lambda, \delta}^{k}[f(s z)]-D_{\lambda, \delta}^{k}[f(t z)]} \\
& =\phi(w(z))=1+b_{1} z+b_{2} z^{2}+\cdots
\end{aligned}
$$

From (2.1), we obtain

$$
a_{2}=\frac{b_{1}}{(1+\lambda)^{k}(\delta+1)(2-s-t)}
$$

and

$$
a_{3}=2 \frac{b_{2}-(s+t)(s+t-2)(1+\lambda)^{2 k}(\delta+1)^{2} a_{2}^{2}}{\left[3-\left(s^{2}+s t+t^{2}\right)\right](1+2 \lambda)^{k}(\delta+1)(\delta+2)}
$$

since

$$
p_{1}(z)=\frac{1+\phi^{-1}(p(z))}{1-\phi^{-1}(p(z))}
$$

then

$$
p(z)=\phi\left[\frac{p_{1}(z)-1}{p_{1}(z)+1}\right]
$$

and

$$
\begin{aligned}
1 & +b_{1} z+b_{2} z^{2}+\cdots=\phi\left(\frac{c_{1} z+c_{2} z^{2}+\cdots}{2+c_{1} z+c_{2} z^{2}+\cdots}\right) \\
& =\phi\left[\frac{1}{2} c_{1} z+\frac{1}{2}\left(c_{2}-\frac{1}{2} c_{1}^{2}\right) z^{2}+\cdots\right]
\end{aligned}
$$

while equating the coefficients of $z$ and $z^{2}$, we infer

$$
b_{1}=\frac{1}{2} B_{1} c_{1} \quad \text { and } \quad b_{2}=\frac{1}{2} B_{1}\left(c_{2}-\frac{1}{2} c_{1}^{2}\right)+\frac{1}{4} B_{2} c_{1}^{2}
$$

From (2.2) and (2.4), we get

$$
\begin{aligned}
a_{2}= & \frac{B_{1} c_{1}}{2(1+\lambda)^{k}(\delta+1)(2-s-t)} \\
\text { and } \quad a_{3}= & \frac{B_{1}}{(1+2 \lambda)^{k}(\delta+1)(\delta+2)\left[3-\left(s^{2}+s t+t^{2}\right)\right]} \\
& \left\{c_{2}-c_{1}^{2}\left(1-\frac{1}{2} \frac{B_{2}}{B_{1}}+\frac{1}{2} B_{1}\left(\frac{s+t}{s+t-2}\right)\right)\right\}
\end{aligned}
$$

Therefore we have

$$
\begin{array}{r}
\left|a_{3}-\mu a_{2}^{2}\right|=\frac{B_{1}}{\left[3-\left(s^{2}+s t+t^{2}\right)\right](1+2 \lambda)^{k}(\delta+1)(\delta+2)} \\
\left\{c_{2}-c_{1}^{2}\left(1-\frac{1}{2} \frac{B_{2}}{B_{1}}+\frac{1}{2} B_{1}\left(\frac{s+t}{s+t-2}\right)\right)\right\} \\
-\frac{\mu B_{1}^{2} c_{1}^{2}}{(\delta+1)^{2}(1+\lambda)^{2 k}(2-s-t)} \\
=\frac{B_{1} \quad\left\{c_{2}^{2}\right\}}{\left[3-\left(s^{2}+s t+t^{2}\right)\right](1+2 \lambda)^{k}(\delta+1)(\delta+2)}\{
\end{array}
$$


where

$$
\begin{array}{r}
v=\left[1-\frac{1}{2} \frac{B_{2}}{B_{1}}+\frac{B_{1}}{2}\left(\frac{s+t}{s+t-2}\right)\right. \\
\left.-\frac{\mu B_{1}(\delta+2)(1+2 \lambda)^{k}\left[3-\left(s^{2}+s t+t^{2}\right)\right]}{(\delta+1)(1+\lambda)^{2 k}(2-s-t)^{2}}\right]
\end{array}
$$

If $\mu \leq \sigma_{1}$, using Lemmas 1.1 and 1.2 , the following is obtained.

$$
\begin{aligned}
\left|a_{3}-\mu a_{2}^{2}\right| & \\
\leq & \frac{4(\delta+1)(\delta+2)(1+2 \lambda)^{k}\left[3-\left(s^{2}+s t+t^{2}\right)\right]}{B_{1}^{2}} \\
& {\left[\left(B_{2}-B_{1}\right)-\frac{B_{1}^{2}}{2}\left(\frac{s+t}{s+t-2}\right.\right.} \\
& \left.\left.+\frac{2 \mu(\delta+2)(1+2 \lambda)^{k}\left[\left(s^{2}+s t+t^{2}\right)-3\right]}{(\delta+1)(1+\lambda)^{2 k}(2-s-t)^{2}}\right)\right]
\end{aligned}
$$

which is the first part of Theorem 1.1.

Similarly, if $\mu \geq \sigma_{2}$, using Lemmas 1.1 and 1.2 , we get $\left|a_{3}-\mu a_{2}^{2}\right|$

$$
\begin{array}{r}
\leq \frac{4(\delta+1)(\delta+2)(1+2 \lambda)^{k}\left[3-\left(s^{2}+s t+t^{2}\right)\right]}{B_{1}^{2}} \\
{\left[\left(B_{1}-B_{2}\right)+\frac{B_{1}^{2}}{2}\left(\frac{s+t}{s+t-2}\right.\right.} \\
\left.\left.+\frac{2 \mu(\delta+2)(1+2 \lambda)^{k}\left[\left(s^{2}+s t+t^{2}\right)-3\right]}{(\delta+1)(1+\lambda)^{2 k}(2-s-t)^{2}}\right)\right]
\end{array}
$$

when $\sigma_{1} \leq \mu \leq \sigma_{2}$, we see that

$$
\begin{aligned}
\left|a_{3}-\mu a_{2}^{2}\right|= & \frac{2 B_{1}\left\{c_{2}-v c_{1}^{2}\right\}}{2(1+2 \lambda)^{k}(\delta+1)(\delta+2)\left(3-\left[s^{2}+s t+t^{2}\right]\right)} \\
& \leq \frac{2 B_{1}}{\delta+1(\delta+2)(1+2 \lambda)^{k}\left[3-\left(s^{2}+s t+t^{2}\right)\right]}
\end{aligned}
$$

Further, if $\sigma_{1} \leq \mu \leq \sigma_{3}$, then

$$
\begin{aligned}
& \left|a_{3}-\mu a_{2}^{2}\right|+\left(\mu-\sigma_{1}\right)\left|a_{2}^{2}\right| \\
& \quad \leq \frac{2 B_{1}}{\delta+1(\delta+2)(1+2 \lambda)^{k}\left[3-\left(s^{2}+s t+t^{2}\right)\right]}
\end{aligned}
$$

Finally,we see that

If $\sigma_{3} \leq \mu \leq \sigma_{2}$, then

$$
\begin{aligned}
& \left|a_{3}-\mu a_{2}^{2}\right|+\left(\sigma_{2}-\mu\right)\left|a_{2}^{2}\right| \\
& \quad \leq \frac{2 B_{1}}{\delta+1(\delta+2)(1+2 \lambda)^{k}\left[3-\left(s^{2}+s t+t^{2}\right)\right]}
\end{aligned}
$$

In order to express that the bounds are sharp, the function $k_{n}^{\phi}(n=2,3, \ldots)$ defined by

$$
\frac{z\left(D_{\lambda, \delta}^{k} k_{n}^{\phi}(z)\right)^{\prime}}{D_{\lambda, \delta}^{k} k_{n}^{\phi}(z)}=\phi\left(z^{n-1}\right), k_{n}^{\phi}(0)=0=\left(k_{n}^{\phi}(0)\right)^{\prime}-1
$$

and the function $F_{\gamma}$ and $G_{\gamma}(0 \leq \gamma \leq 1)$ by

$$
\frac{z\left(D_{\lambda, \delta}^{k} F_{\gamma}(z)\right)^{\prime}}{D_{\lambda, \delta}^{k} F_{\gamma}(z)}=\phi\left(\frac{z(z+\gamma)}{1+\gamma z}\right), F_{\gamma}(0)=0=\left(F_{\gamma}(0)\right)^{\prime}-1
$$

and

$$
\frac{z\left(D_{\lambda, \delta}^{k} G_{\gamma}(z)\right)^{\prime}}{D_{\lambda, \delta}^{k} G_{\gamma}(z)}=\phi\left(-\frac{z(z+\gamma)}{1+\gamma z}\right), G_{\gamma}(0)=0=\left(G_{\gamma}(0)\right)^{\prime}-1
$$

Clearly the functions $k_{n}^{\phi}, F_{\gamma}$ and $G_{\gamma} \in R_{\lambda, \delta}^{k}(\phi)$. It can also be denoted as $K^{\phi}=K_{2}^{\phi}$. If $\mu<\sigma_{1}$ or $\mu>\sigma_{2}$, then the equality in Theorem 2.1 satisfies iff $f$ is $K^{\phi}$ or one of its rotations.

If $\sigma_{1}<\mu<\sigma_{2}$, then the equality satisfies iff $f$ is $K_{3}^{\phi}$ or one of its rotations.

When $\mu=\sigma_{1}$, the equality satisfies iff $f$ is $F_{\gamma}$ or one of its rotations. When $\mu=\sigma_{2}$, the equality satisfies iff $f$ is $G_{\gamma}$ or one of its rotations.

Using Lemma 1.2,the following theorem can easily be obtained.

Theorem 2.2. Let $\phi(z)=1+B_{1} z+B_{2} z^{2}+\cdots$, where $B_{n}$ are real with $B_{1}>0$ and $B_{2} \geq 0$. If $f(z)$ given by (1.1) belongs to $R_{\lambda, \delta}^{k}(\phi)$, then

$\left|a_{3}-\mu a_{2}^{2}\right|$

$$
\begin{array}{r}
\leq\left(\frac{4 B_{1}}{(1+2 \lambda)^{k}(\delta+1)(\delta+2)\left[3-\left(s^{2}+s t+t^{2}\right)\right]}\right) \\
\times \max \left\{1, \mid 1-\frac{2 B_{2}}{B_{1}}+B_{1}\left(\frac{s+t}{s+t-2}\right.\right. \\
\left.\left.-\frac{2 \mu(\delta+2)(1+2 \lambda)^{k}\left[3-\left(s^{2}+s t+t^{2}\right)\right]}{(\delta+1)(2-s-t)^{2}(1+\lambda)^{2 k}}\right) \mid\right\},
\end{array}
$$

$\mu \in \mathbb{C}$. The result is Sharp.

Remark 2.3. The coefficient bounds for $\left|a_{2}\right|$ and $\left|a_{3}\right|$ are special cases of those claimed by Theorem 2.1

Remark 2.4. In its distinctive case when $\lambda=1, \delta=0$ and $k=0$, a known result of Ma and Minda [9] was arrived.

Remark 2.5. In its distinctive case, when $\lambda=1, \delta=0$ and $k=0, s=1, t=-1$, a known result due to T.N Shanmugam et al [17] was arrived.

\section{Applications to Analytic Function Defined by Fractional Calculus}

The dependece of fractional calculus has earned appreciable demand upon early decades. Two of the current contributions on this area of deeprooted investigations include comprehensive treatises on the theory and applications of fractional differential equations by Podlubny [13] and Kilbas et al.[5]

We first introduce the class $\mathscr{M}_{\alpha, \beta, \lambda}^{\delta}(\phi)$, that is defined using Hadamard product and a certain operator Owa-Srivatsava operator (see for details, [18] and [8] ; see also [11], [12] and [21] ) in fractional calculus. 
Definition 3.1. The fractional integral of order $\delta$ is elucidated for $f(z)$,

$$
D_{z}^{-\delta} f(z)=\frac{1}{\Gamma(\delta)} \int_{o}^{z} \frac{f(\zeta)}{(z-\zeta)^{1-\delta}} d \zeta \quad(\delta>0)
$$

where $f(z)$ is analytic in a simply-connected domain of the complex $z$-plane that contains the origin and the multiplicity of $(z-\zeta)^{\delta-1}$ is expelled by including $\log (z-\zeta)$ to be real when $z-\zeta>0$

Definition 3.2. The fractional derivative of order $\delta$ is elucidated, for $f(z)$

$$
D_{z}^{\delta} f(z)=\frac{1}{\Gamma(1-\delta)} \int_{o}^{z} \frac{f(\zeta)}{(z-\zeta)^{\delta}} d \zeta \quad(0 \leq \delta<1)
$$

where $f(z)$ is constrained, and the multiplicity of $(z-\zeta)^{-\delta}$ is expelled by requiring $\log (z-\zeta)$ to be real when $z-\zeta>0$

Definition 3.3. Using Definition 3.2, the fractional derivative of order $n+\delta$ is elucidated, for $f(z)$

$$
D_{z}^{n+\delta} f(z)=\frac{d^{n}}{d z^{n}}\left\{D_{z}^{\delta} f(z)\right\} \quad\left(0 \leq \delta<1 ; n \in \mathbb{N}_{0}\right)
$$

Using Definitions 3.1, 3.2 and 3.3 of fractional derivatives and fractional integrals, Owa and Srivatsava put forward the Owa-Srivatsava operator

$$
\left(\Omega^{\delta} f\right)(z)=\Gamma(2-\delta) z^{\delta} D_{z}^{\delta} f(z), \quad(\delta \neq 2,3,4, \ldots)
$$

In terms of the Owa-Srivatsava operator $\Omega^{\delta}$ defined by 3.4 , we now introduce the function class $\mathscr{M}_{\alpha, \beta, \lambda}^{\delta}(\phi)$ in the following way:

$$
\mathscr{M}_{\alpha, \beta, \lambda}^{\delta}(\phi)=\left\{f: f \in \mathscr{A} \text { and } \Omega^{\delta} f \in \mathscr{M}_{\alpha, \beta, \lambda}^{\delta}(\phi)\right\} .
$$

Evidently, the function class $\mathscr{M}_{\alpha, \beta, \lambda}^{\delta}(\phi)$ is a special case of the function class $\mathscr{M}_{\alpha, \beta, \lambda}^{g}(\phi)$ if

$$
g(z)=z+\sum_{n=2}^{\infty} \frac{\Gamma(n+1) \Gamma(2-\delta)}{\Gamma(n+1-\delta)} z^{n}
$$

Suppose

$$
g(z)=z+\sum_{n=2}^{\infty} g_{n} z^{n} \quad\left(g_{n}>0\right)
$$

since

$$
f(z)=z+\sum_{n=2}^{\infty} a_{n} z^{n} \in \mathscr{M}_{\alpha, \beta, \lambda}^{\delta}(\phi)
$$

if and only if

$$
(f * g)(z)=z+\sum_{n=2}^{\infty} g_{n} a_{n} z^{n} \in \mathscr{M}_{\alpha, \beta, \lambda}(\phi)
$$

the coefficient estimates are obtained for the functions in the class $\mathscr{M}_{\alpha, \beta, \lambda}^{g}(\phi)$ with respect to the corresponding estimates for functions in the class $\mathscr{M}_{\alpha, \beta, \lambda}(\phi)$. Using Theorem 2.1 to the following Hadamard product:

$$
(f * g)(z)=z+g_{2} a_{2} z^{2}+g_{3} a_{3} z^{3}+\ldots,
$$

we infer the Theorem 3.4 defined below after an evident change of the parameter $\mu$.

Theorem 3.4. Let

$$
0 \leq \mu \leq 1,0 \leq \alpha \leq 1,0<\beta \leq 1 \text { and } 0 \leq \lambda \leq 1 .
$$

Suppose

$$
\phi(z)=1+B_{1} z+B_{2} z^{2}+B_{3} z^{3}+\ldots,
$$

where the coefficients $B_{n}$ are real with

$$
B_{1}>0, B_{2}>0 \text {, and } B_{n}>0 \quad(n \in \mathbb{N} \backslash\{1,2\}) .
$$

When $f(z)$ given by (1.1) be the part of the class $\mathscr{M}_{\alpha, \beta, \lambda}^{g}(\phi)$, then

$$
\left|a_{3}-\mu a_{2}^{2}\right| \leq \begin{cases}\frac{\Lambda}{g_{3}}, & \text { if } \mu \leq \sigma_{5} \\ \frac{\eta}{g_{3}}, & \sigma_{5} \leq \mu \leq \sigma_{6} \\ -\frac{\Lambda}{g_{3}}, & \mu \geq \sigma_{6} .\end{cases}
$$

where

$$
\begin{aligned}
\sigma_{5}= & \frac{g_{3}}{g_{2}^{2}} \frac{(\delta+1)(s+t-2)(1+\lambda)^{2 k}}{2(\delta+2)(1+2 \lambda)^{k}\left[\left(s^{2}+s t+t^{2}\right)-3\right]} \\
& {\left[\frac{2\left(B_{2}-B_{1}\right)(s+t-2)-B_{1}^{2}(s+t)}{B_{1}^{2}}\right] } \\
\sigma_{6}= & \frac{g_{3}}{g_{2}^{2}} \frac{(\delta+1)(s+t-2)(1+\lambda)^{2 k}}{2(\delta+2)(1+2 \lambda)^{k}\left[\left(s^{2}+s t+t^{2}\right)-3\right]} \\
& {\left[\frac{2 B_{2}(s+t-2)-(s+t) B_{1}^{2}}{B_{1}^{2}}\right] }
\end{aligned}
$$

where $\Lambda$ and $\eta$ are elucidated in Theorem 2.1, respectively. These results are sharp. Since, by (1.1) and 3.4,

$$
\left(\Omega^{\delta} f\right)(z)=z+\sum_{n=2}^{\infty} \frac{\Gamma(n+1) \Gamma(2-\delta)}{\Gamma(n+1-\delta)} a_{n} z^{n}
$$

we readily obtain

$$
g_{2}=\frac{\Gamma(3) \Gamma(2-\delta)}{\Gamma(3-\delta)}=\frac{2}{2-\delta}
$$

and

$$
g_{3}=\frac{\Gamma(4) \Gamma(2-\delta)}{\Gamma(4-\delta)}=\frac{6}{(2-\delta)(3-\delta)} .
$$

For $g_{2}$ and $g_{3}$ obtained by (3.9) and (3.10), respectively, Theorem 3.4 diminishes to the interesting result. 
Theorem 3.5. Let

$$
0 \leq \mu \leq 1,0 \leq \alpha \leq 1,0<\beta \leq 1 \text { and } 0 \leq \lambda \leq 1
$$

Suppose also that

$$
\phi(z)=1+B_{1} z+B_{2} z^{2}+B_{3} z^{3}+\ldots,
$$

where the coefficients $B_{n}$ are real with

$$
B_{1}>0, B_{2}>0, \text { and } B_{n}>0(n \in \mathbb{N} \backslash\{1,2\}) \text {. }
$$

If $f(z)$ given by (1.1) belongs to the class $\mathscr{M}_{\alpha, \beta, \lambda}^{g}(\phi)$, then

$$
\left|a_{3}-\mu a_{2}^{2}\right| \leq \begin{cases}\frac{(2-\delta)(3-\delta)}{6} \Lambda, & \text { if } \mu \leq \sigma_{7} \\ \frac{(2-\delta)^{6}(3-\delta)}{6} \eta, & \sigma_{7} \leq \mu \leq \sigma_{8} \\ -\frac{(2-\delta)(3-\delta)}{6} \Lambda, & \mu \geq \sigma_{8}\end{cases}
$$

where, for convenience,

$$
\begin{array}{r}
\sigma_{7}=\frac{2(3-\delta)}{3(2-\delta)} \frac{g_{3}}{g_{2}^{2}} \frac{(\delta+1)(s+t-2)(1+\lambda)^{2 k}}{2(\delta+2)(1+2 \lambda)^{k}\left[\left(s^{2}+s t+t^{2}\right)-3\right]} \\
{\left[\frac{2\left(B_{2}-B_{1}\right)(s+t-2)-B_{1}^{2}(s+t)}{B_{1}^{2}}\right]} \\
\sigma_{8}=\frac{2(3-\delta)}{3(2-\delta)} \frac{(\delta+1)(s+t-2)(1+\lambda)^{2 k}}{2(\delta+2)(1+2 \lambda)^{k}\left[\left(s^{2}+s t+t^{2}\right)-3\right]} \\
{\left[\frac{2 B_{2}(s+t-2)-(s+t) B_{1}^{2}}{B_{1}^{2}}\right]}
\end{array}
$$

and $\Lambda$ and $\eta$ are defined as in Theorem 2.1, respectively.

Remark 3.6. In its special case, when

$$
\lambda=0, \beta=1 \alpha=0, B_{1}=\frac{8}{\pi^{2}} \text { and } B_{2}=\frac{16}{3 \pi^{2}}
$$

Theorem 3.5 coincides with the following result due to Srivatsava et al. [19] for which $\Omega^{\lambda} f(z)$ is a parabolic starlike function ([3] and [16]).

Remark 3.7. When

$$
\lambda=0, \beta=1 \alpha=0, \delta=1, B_{1}=\frac{8}{\pi^{2}} \text { and } B_{2}=\frac{16}{3 \pi^{2}}
$$

Theorem 3.5 would coincide with the result obtained earlier by Ma and Minda [10]

\section{References}

[1] M.Darus and K.Al-shaqsi, Differential Sandwich theorem with generalized derivative operator, Proc.World Acad. Sci. Eng. Tech. , 28 (2008),11-14.

[2] M.Fekete and G.szegö, Eine Bermerkung ber ungerade schlichte Funktionen, J.Lond.Math.Soc., 8 (1933), 85-89.
[3] A.W.Goodman, Uniformly Convex Functions, Ann. Polon. Math., 56 (1991), 87-92.

[4] F.R.Keogh and E.P.Merkes, A Coefficient inequality for certain classes of analytic functions, Proc.Amer.Math.Soc., 20 (1969), 8-12.

[5] A.A.Kilbas, H.M.Srivatsava and J.J.Trujillo, Theory and Applications of Fractional Differential Equations, North Holland Mathematics Studies, Elsevier (North-Holland) Science Publishers, Amsterdam, London, New York, 204 (2006).

[6] W.Koepf, On the Fekete-szegö problem for Close-toconvex functions II, Arch.Math., 49 (1987), 490-533.

[7] W.Koepf, On the Fekete-szegö problem for Close-toconvex functions II, Proc.Amer.Math.Soc., 101 (1987), 89-95.

[8] S.Owa, An Application of the Fractional Derivative,Math. Japan., 29 (1984), 383-389.

[9] W. Ma and D. Minda, A Unified treatment of some special classes of univalent functions, in Proc. Conf. on Complex Analysis(Tianjin,1992), 157-169, Conf. Proc. Lecture Notes Anal., Vol. I, Int. Press, Cambridge,MA.

[10] W. Ma and D. Minda,Uniformly Convex Functions.II, Ann. Polon. Math.,58(1993), 275-285.

[11] S.Owa, On the Distortion Theorems, I,Kyungpook Math.J., 18 (1978), 53-59.

[12] S.Owa and H.M.Srivatsava, Univalent and Starlike Generalized Hypergeometric Functions, Canad. J.Math., 39 (1987), 1057-1077.

[13] I.Podlubny, Fractional Differential Equations:An Introduction to Fractional Derivatives, Fractional Differential Equations, Methods of their solutions and some of their Applications, Mathematics in Science and Engineering, Academic Press, New York, London and Toronto, 198 (1999).

[14] C.Ramachandran, S.Sivasubramanian, H.M.Srivatsava and A.Swaminathan, Coefficient inequalities for certain subclasses of analytic functions and their applications involving the Owa-Srivatsava operator of fractional calculus, J.Math. Inequal. Appl. 12 (2) (2009), 351-363.

[15] V Ravichandran, Metin Bolcal, Yasar Polotoglu and A. Sen, Certain Subclasses of Starlike and Convex functions of complex order, Hacettepe J. Math. and Stat., 34 (2005), 9-15.

[16] F.Ronning, Uniformly Convex Functions and a corresponding class of starlike Functions, Proc. Amer. Math. Soc.118 (1993), 189-196.

[17] T.N.Shanmugam, C.Ramachandran and V.Ravichandran, "Fekete-szegöProblem for Subclass of Starlike Functions With Respect to Symmetric Points", Bull. Korean Math. Soc., 43(3) (2006), 589-598.

[18] H.M.Srivatsava, Some families of fractional derivative and other linear operators associated with analytic, univalent and multivalent functions, in Analysis and its applications(Chennai 2000), Allied Publ. Ltd., New Delhi, Mumbai, Calcutta and Chennai, (2001), 209-243. 
[19] H.M.Srivatsava and A.K.Mishra, Applications of Fractional Calculus to Parabolic Starlike and Uniformly Convex Functions, Comput. Math Appl. 39(3-4) (2000), 5769.

[20] H.M.Srivatsava, A.K.Mishra and M.K.Das, the Feketeszegö problem for a subclass of Close-to-convex functions, Complex Variables Theory Appl., 44 (2001), 145163.

[21] H.M.Srivatsava and S.Owa, Univalent Functions, Fractional Calculus and their Applications, Halsted Press(Ellis Horwood Ltd., Chichester), John Wiley and Sons, New York, Chichester, Brisbane and Toronto, (1989). 